\title{
Sleep Apnea and Diabetes Predict Progression of Cardiac Diastolic Dysfunction in Pre-heart Failure Patients: a Prospective Cohort HSCAA Study
}

\section{Yonekazu Kidawara}

Hyogo College of Medicine: Hyogo Ika Daigaku

Manabu Kadoya

Hyogo College of Medicine: Hyogo lka Daigaku

\section{Akiko Morimoto}

Hyogo College of Medicine: Hyogo Ika Daigaku

\section{Takashi Daimon}

Hyogo College of Medicine: Hyogo Ika Daigaku

Miki Kakutani-Hatayama

Hyogo College of Medicine: Hyogo Ika Daigaku

Kae Kosaka-Hamamoto

Hyogo College of Medicine: Hyogo Ika Daigaku

Akio Miyoshi

Hyogo College of Medicine: Hyogo Ika Daigaku

Kosuke Konishi

Hyogo College of Medicine: Hyogo Ika Daigaku

Yoshiki Kusunoki

Hyogo College of Medicine: Hyogo Ika Daigaku

Takuhito Shoji

Hyogo College of Medicine: Hyogo Ika Daigaku

\section{Akiko Goda}

Hyogo College of Medicine: Hyogo Ika Daigaku

\section{Masanori Asakura}

Hyogo College of Medicine: Hyogo lka Daigaku

\section{Masaharu Ishihara}

Hyogo College of Medicine: Hyogo lka Daigaku

Hidenori Koyama ( $\nabla$ hkoyama@hyo-med.ac.jp )

Department of Diabetes, Endocrinology and Clinical Immunology Hyogo College of Medicine 1-1 Mukogawa-cho, Nishinomiya, Hyogo 663-8501, Japan https://orcid.org/0000-0001-7930-2228 
Keywords: Type 2 diabetes, sleep apnea, diastolic dysfunction, longitudinal analysis

Posted Date: October 2nd, 2020

DOI: https://doi.org/10.21203/rs.3.rs-84378/v1

License: (c) (1) This work is licensed under a Creative Commons Attribution 4.0 International License. Read Full License 


\section{Abstract}

[Background] Sleep apnea, a common co-morbid condition of diabetes, has been shown to be associated with established heart failure. However, its role in association of the presence of diabetes in the progression of cardiac diastolic function in pre-heart failure phase is not known. This prospective study is to longitudinally examine the predictive value of sleep apnea and diabetes on progression of left ventricular (LV) diastolic dysfunction in patients without heart disease.

[Methods] Among 976 patients registered in HSCAA prospective cohort study, 517 without heart disease (175 type 2 diabetes, 342 non-diabetes) were followed with repeated echocardiography in every 1-3 years for a mean 34.7 months. LV diastolic dysfunction was determined by transmitral early inflow velocity/early diastolic tissue velocity $\left(E / e^{\prime}\right)>14$. Annual change in $E / e^{\prime}$ was calculated by using the slope of the linear regression line calculated from at least 3 echocardiographic measurements. Sleep apnea was determined by an apnomonitor device in conjunction with percutaneous oxygen saturation, and apnea hypopnea index (AHI) was calculated.

[Results] Kaplan-Meier analyses revealed that subjects with diabetes or sleep apnea had a significantly $(p<0.01)$ greater risk for LV diastolic dysfunction, with hazards ratio of 2.21 (1.41-3.47) and 2.23 (1.403.55), respectively. Subjects with sleep apnea exhibited significantly higher risk for LV diastolic dysfunction in non-diabetic subjects $(p<0.01)$, while showed tendency of higher risk in diabetic subjects $(p=0.10)$. The annual change of $E / e^{\prime}$ was significantly and independently associated with both diabetes $(\beta=0.278, p<0.01)$ and $A H I(\beta=0.138, p<0.01)$. Finally, ROC analyses revealed that addition of $A H I$ and diabetes to classical risk factors best predicted individuals with fast progression of diastolic dysfunction (annual change of $E / e^{\prime}>1.0$ ) with an AUC of 0.81 .

[Conclusions] In patients without heart disease, sleep apnea is an important predictor for progression of LV diastolic dysfunction. Its association is partly confounded, but still independent of the presence of diabetes.

\section{Introduction}

Diabetic cardiomyopathy is defined by abnormal myocardial structure and performance in the absence of coronary artery disease, hypertensive cardiomyopathy, and valvular heart disease, in individuals with diabetes mellitus [1]. In early stages, diabetic cardiomyopathy exhibits subclinical structural and functional abnormalities, including left ventricular (LV) hypertrophy and fibrosis, and these pathophysiological changes are associated with subclinical diastolic dysfunction that often progresses to heart failure (HF) with preserved ejection fraction (HFpEF) [2, 3].

In addition to hyperglycemic burden, the risk of HFpEF has been shown to sharply increase in the presence of other risk factors [4]. Obstructive sleep apnea, a frequent comorbid condition noted in diabetic patients [5], is commonly observed in association with heart failure and may be attributable to worsening of the disease [6]. Sleep problems including sleep apnea have also been shown to be closely 
associated with increased incidence of HFpEF [7]. Thus, mutual interactions of diabetes and sleep apnea may contribute to progression of diabetes cardiomyopathy, particularly diastolic dysfunction. However, integrated predictors including these specific factors have yet to be investigated, which is important for demonstrating progression of left ventricular diastolic dysfunction in pre-heart failure patients.

In this prospective cohort study, we examined the impact of sleep apnea and diabetes as predictors of progression of cardiac diastolic dysfunction in pre-heart failure patients registered in the Hyogo Sleep Cardio-Autonomic Atherosclerosis (HSCAA) study.

\section{Methods}

\section{DESIGN AND STUDY PARTICIPANTS}

The HSCAA study is an ongoing single center cohort performed for a review of cardio-metabolic risk factors, including quantitatively measured sleep parameters, for elucidation of the clinical implications of cardiovascular and metabolic diseases [8]. From October 2010 to December 2018, 976 patients with at least 1 cardiovascular risk factor, including obesity, hypertension, dyslipidemia, diabetes mellitus, and chronic kidney diseases, were enrolled in that cohort study. Patients $(n=240)$ with ischemic heart disease, moderate to severe valvular heart disease, hypertrophic cardiomyopathy, atrial fibrillation, HF, or cardiac diastolic dysfunction [transmitral early inflow velocity/early diastolic tissue velocity (E/e' $>14)$ ], assessed according to the recommendations of the American Society of Echocardiography and European Association of Cardiovascular Imaging [9] were excluded. After excluding another 131 with missing baseline data for cardiac ultrasonography and 88 with a follow-up period $<12$ months, 517 of those patients were enrolled in the present study (Figure 1). A large majority of the participants $(n=462)$ underwent apnomonitor monitoring for a review of sleep apnea. The HSCAA study was approved by an appropriate institutional ethical committee (approval No. 2351) and informed written consent was obtained from each participant.

\section{ASSESSMENT OF CLASSICAL CARDIOVASCULAR RISK FACTORS}

Body mass index (BMI) was calculated as weight in kilograms divided by the square of height in meters $\left(\mathrm{kg} / \mathrm{m}^{2}\right)$. Smoking status was based on self-reported cigarette smoking habit. Blood pressure and pulse rate were measured twice in a sitting position after a deep breath, with the mean values used for analysis. Type 2 diabetes was diagnosed based on results showing fasting plasma glucose $\geq 126 \mathrm{mg} / \mathrm{dl}$, causal plasma glucose $\geq 200 \mathrm{mg} / \mathrm{dl}$, or 2-hour plasma glucose $\geq 200 \mathrm{mg} / \mathrm{dl}$ during a $75-\mathrm{g}$ oral glucose tolerance test, or previous therapy for diabetes [10]. Hypertension was defined as systolic $B P \geq 140 \mathrm{mmHg}$, diastolic $\mathrm{BP} \geq 90 \mathrm{mmHg}$, or treatment for hypertension. Dyslipidemia was defined based on results showing low density lipoprotein $(L D L)$ cholesterol $(\geq 140 \mathrm{mg} / \mathrm{dl})$, high density lipoprotein (HDL) cholesterol $(\leq 40$ $\mathrm{mg} / \mathrm{dl})$, elevated triglyceride level ( $\geq 150 \mathrm{mg} / \mathrm{dl})$, or treatment for dyslipidemia.

\section{ASSESSMENT OF LV DIASTOLIC FUNCTION}


LV function was evaluated by echocardiography as previously described [11]. Examinations were repeated every 1-3 years to determine changes in LV function. To measure LV ejection fraction (LVEF), we used the modified Simpson's method for patients with LV segmental asynergy or deformation, and Teichholz's formula for patients without LV asynergy or deformation. LV mass index (LVMI) was calculated as follows: $\quad$ LVMI $=1.04 \times\left\{(\text { LVDd + IVST + RWT })^{3}-(\text { LVDd })^{3}-13.6\right\} / 1000 / B S A$, in which RWT (relative wall thickness) $=$ IVST + PWT/LVDd, BSA (body surface area) $=$ height $(\mathrm{cm})^{0.725} \times$

weight $(\mathrm{kg})^{0.425} \times 0.7184$ (LVDd: left ventricular diastolic dimension, IVST: intraventricular septal thickness, PWT: posterior wall thickness). Transmitral early inflow velocity (E-wave), late diastolic filling velocity (A-wave), and E-wave deceleration time (DcT) were determined using pulsed Doppler echocardiography [9]. Early diastolic tissue velocity (e') was measured in the septal basal region using tissue Doppler imaging and the E/e' ratio was then calculated to obtain estimated LV filling pressure. Among these parameters, E/e' $>14$ was evaluated as LV diastolic dysfunction as recommended [9]. Annual change in E/e' was estimated by using the slope (b) of the linear regression line calculated from at least 3 echocardiographic measurements with the following equation: $E / e^{\prime}=a+b \times$ months.

\section{ASSESSMENT OF SLEEP APNEA}

To examine the presence of sleep apnea, we used an apnomonitor device (SAS-2100 ${ }^{\circledR}$, Teijin, Tokyo, Japan) to determine apnea hypopnea index (AHI), as previously described [8]. Percutaneous oxygen saturation was recorded using a pulse oximeter. Apnea was defined as complete cessation of air flow lasting $\geq 10$ seconds, hypopnea as a $\geq 50 \%$ reduction in air flow lasting $\geq 10$ seconds associated with a $4 \%$ decrease in oxygen saturation, and $\mathrm{AHI}$ as the average number of apnea and hypopnea episodes per hour.

\section{PLASMA BIOCHEMICAL PARAMETERS}

Blood samples were obtained in the morning after an overnight fast and then quickly centrifuged to obtain plasma. Whole blood was used for hemoglobin A1c, EDTA-plasma for glucose, and lipids, and serum for other biochemical assays. Glucose was measured using a glucose oxidase method. Insulin was determined by chemiluminesce enzyme immunoassay results and serum creatinine concentration with an enzymatic method. Estimated glomerular filtration rate (eGFR) in each subject was calculated using an equation for Japanese subjects, as follows: eGFR $\left(\mathrm{ml} / \mathrm{min} / 1.73 \mathrm{~m}^{2}\right)=$ $194 \times$ age $(\text { years })^{-0.287} \times$ S-creatinine ${ }^{-1.094}$ (if female, $\times 0.739$ ).

\section{STATISTICAL ANALYSES}

To compare mean and median values among the groups, Student's t-test and a Mann-Whitney test were used. A chi-square test was used for comparisons for dichotomous variables. To analyze associations between factors, parameters with skewed distribution were natural logarithm-transformed (In) to normalize skewed distribution. For this study, the primary outcome was LV diastolic dysfunction, which as defined as $E / e^{\prime}>14$, as previously recommended [9]. Outcome rates for the groups were compared 
using Kaplan-Meier analysis and a log-rank test. Prognostic variables for primary outcome were examined using a Cox proportional hazards regression model. Predictors for annual changes of E/e' were examined using Pearson's correlation coefficient and logistic regression analysis. To compare each logistic model in aggregate, we constructed receiver operating characteristic (ROC) curves and determined the area under the curve (AUC). All statistical analyses were performed using the Statistical Package for Social Sciences software package (PASW Statistics version 18.0). All reported $p$ values are 2tailed and were considered statistically significant at $<0.05$.

\section{Results}

\section{Subjects}

Among 976 patients registered in the HSCAA cohort study, 517 (342 non-diabetic, 175 diabetic) were prospectively followed, including echocardiographic evaluations repeated every 1-3 years (Figure 1). The mean follow-up period was $34.7 \pm 18.0$ (standard deviation) months. Table 1 summarizes basal clinical characteristics of the enrolled subjects who were categorized into the diabetic and non-diabetic groups. As compared with the non-diabetic subjects, the diabetic group exhibited higher age, male prevalence, higher body mass index, more current smokers, higher systolic BP, lower LDL- and HDL-cholesterol, higher fasting plasma glucose and $\mathrm{HbA} 1 \mathrm{c}$, and a higher number with comorbidity of dyslipidemia, more likely to use statin and renin-angiotensin system inhibitors. $\mathrm{AHI}$ and prevalence of a score $>10$ were significantly higher in the diabetic group (Table 1).

\section{Predictors of events of cardiac diastolic dysfunction ( $\left.E / e^{\prime}>14\right)$}

As shown in Table 1, the majority of parameters examined for $L V$ diastolic function (A wave, E/A, e', E/e') were significantly worse in the diabetic group. LVEF was identical between the groups, while LVMI tended to be higher in the diabetic than non-diabetic subjects. During the follow-up period, cardiac diastolic dysfunction ( $\left.E / e^{\prime}>14\right)$ events were more frequently observed in the diabetic subjects (Table 1$)$ and Kaplan-Meier analysis results revealed that the event (E/e' $>14)$-free survival rate was also significantly lower in that group (Figure 2A) with hazards ratio (HR) of 2.21 (1.41-3.47 95\% confidence interval). In all subjects, those with sleep apnea $(\mathrm{AHI}>10)(\mathrm{p}<0.01)$ with HR 2.23 (1.40-3.55) exhibited higher events of cardiac diastolic dysfunction as compared to subjects without that condition (Figure 2A). In the nondiabetic group, subjects with sleep apnea had a significantly greater number of diastolic dysfunction events (Figure 2B), while in the diabetic group, sleep apnea tended to be associated with higher rate of diastolic dysfunction events (Figure 2C).

\section{Predictors of annual change in E/e'}

Baseline E/e' markedly affected events of cardiac diastolic dysfunction (HR 1.36, 95\% Cl 1.25-1.48), thus we analyzed clinical factors associated with annual change in $\mathrm{E} / \mathrm{e}^{\prime}$ based on results of multiple $(\geq 3$ times) echocardiographic measurements. In accordance with the results shown in Figure 2, diabetes and AHI were significantly and positively associated with annual change in $\mathrm{E} / \mathrm{e}^{\prime}$ in all subjects (Table $2 \mathrm{~A}$ ). 
Baseline $\mathrm{E} / \mathrm{e}^{\prime}$ was strongly and inversely associated with annual $\mathrm{E} / \mathrm{e}^{\prime}$ change, suggesting blunted $\mathrm{E} / \mathrm{e}^{\prime}$ progression in patients with already high E/e'. Multiple linear regression analyses in all subjects showed that both diabetes and $\mathrm{AHI}$ were significantly independent and positive predictors of annual progression of E/e' (Table 2B).

To further examine the impact of diabetes and $\mathrm{AHI}$ on prediction of diastolic dysfunction progression, we performed multiple logistic regression analyses to predict fast progression of diastolic dysfunction, which was determined by annual change in $\mathrm{E} / \mathrm{e}^{\prime}>1.0$. As shown in Figure $3,14.4 \%$ of all subjects exhibited an annual change in $E / e^{\prime}>1.0$, while the percentage was $22.7 \%$ in the diabetic and $9.7 \%$ in the non-diabetic group. As seen in the upper panel of Figure 4, ROC analysis revealed that addition of diabetes to basal models including age, gender, $\mathrm{BMI}$, hypertension, dyslipidemia and baseline $\mathrm{E} / \mathrm{e}^{\prime}$ resulted in a significant $(p=0.04)$ increase in AUC from 0.73 to 0.77 , whereas addition of AHI (0.75) instead of diabetes to the basal model (0.73) did not significantly change AUC. When AHI was further added to basal + diabetes, AUC was improved up to the highest value of 0.81 , significantly higher than that for the basal model $(p<0.01)$ but not reaching statistically significant than that for basal + diabetes $(p=0.28)$.

\section{Discussion}

This is the first study to examine the impact of sleep apnea as well as diabetes on progression of cardiac diastolic dysfunction in patients without heart failure. Patients with asymptomatic HF based on cardiac ultrasonography findings together with the index of $\mathrm{E} / \mathrm{e}^{\prime}>14$ were carefully excluded from analyses. We found that in addition to the presence of diabetes, sleep apnea was significantly associated with worsening of LV diastolic function. Moreover, addition of sleep apnea index to diabetes resulted in the best prediction of subjects with rapid progression of diastolic dysfunction.

\section{Sleep apnea as predictor of progression of cardiac diastolic dysfunction}

Although sleep problems including obstructive sleep apnea have been demonstrated to be closely associated with increased incidence of HFpEF [7], reports showing an association of sleep apnea with LV diastolic function, such as E/e', in patients without $H F$ are limited. In 206 patients with $A H I \geq 5 / h$ and without cardiac disease, hypertension, or diabetes, AHI was shown to be associated with higher E/e' [12]. Also, in 78 patients with obstructive sleep apnea without any comorbidities, disease severity tended to be associated with both LV diastolic dysfunction and abnormalities in regional systolic function with myocardial deformation changes, in spite of normal LV ejection fraction [13]. In a recent report of 1506 adults in the Hispanic Community Health Study/Study of Latinos ECHO-SOL Ancillary Study, greater AHI was associated cross-sectionally with subclinical markers of LV diastolic dysfunction (E/e') and LVMI [14].

To the best of our knowledge, no longitudinal studies that examined the predictive value of sleep apnea on progression of cardiac diastolic dysfunction are available. In our study, sleep apnea was conclusively shown to be a significant predictor of progression of LV diastolic dysfunction based on multiple results; (1) patients with $\mathrm{AHI}>10$ exhibited significantly greater numbers of diastolic dysfunction events (E/e' 
>14), (2) AHI value was significantly and independently associated with annual progression of $E / e^{\prime}$, and (3) addition of $\mathrm{AHI}$ to classical risk factors including diabetes in ROC analyses best predicted an individual with annual worsening of $\mathrm{E} / \mathrm{e}^{\prime}>1.0$. Interestingly, $\mathrm{AHI}$ was not significantly associated with annual changes in LVMI in all, non-diabetic and diabetic subjects (data not shown), implicating discrete underlying mechanisms involved in the progression of LV hypertrophy and diastolic dysfunction. In addition to diabetes, we consider that sleep apnea is a promising potential predictor for progression of LV diastolic dysfunction.

\section{Diabetes and cardiac diastolic dysfunction}

LV diastolic dysfunction is an important cause of HFpEF in individuals with diabetes and epidemiologic studies have demonstrated the presence of diastolic dysfunction in type 2 diabetes patients $[15,16]$. In our recent study, diabetic patients exhibited higher E/e' as compared to those with either normal or impaired glucose tolerance [11]. Among the available parameters of LV diastolic function, E/e' determined with a non-invasive approach has long been used to assess LV filling pressure in various patient populations [17]. In type 2 diabetes patients, E/e' has been shown to provide independent and incremental prognostic information for predicting cardiovascular morbidity and mortality [18]. Thus, elevated E/e' can be a useful parameter of early diastolic impairment in diabetic patients, potentially foretelling diabetic cardiomyopathy. Longitudinal studies of diabetic patients are needed to better understand and predict this subtle slow onset of diabetic cardiomyopathy, though such evaluations of changes in continuous parameters of LV diastolic function over time in patients without clinically diagnosed HF are scant. Previously, the presence of diabetes was shown to be associated with changes in several cardiac parameters over an 8-year period, including LVEF by $-2.98 \%$, LV mass index by $+5.86 \mathrm{~g}$, and $E / e^{\prime}$ by +1.64 [19]. In that study, LV systolic function and LV mass deteriorated similarly along with E/e' during the 8-year follow-up period. In the present study, frequent measurements of cardiac function showed that diabetic subjects exhibited significantly greater annual changes in E/e' than non-diabetic subjects, whereas LVEF did not show obvious changes. Also, baseline LVMI was not significantly different between the diabetes and non-diabetes groups. On the other hand, annual changes in LVMI were significantly greater in our diabetic subjects, although these changes were not as marked as the changes in E/e' (data not shown). Moreover, baseline LVMI was not significantly associated with annual change in $E / e^{\prime}$ in all subjects $(r=0.20, P=0.73)$, suggesting that $L V$ hypertrophy may not be essential for the progression of E/e'. Future kinetics analyses to compare the progression of E/e' and LVMI in diabetic patients are warranted to clarify the pathological sequence of diabetic cardiomyopathy.

Potential mediators of the association between diabetes and cardiac diastolic dysfunction have yet to be well clarified. A previous study showed that even in a population with normal glucose tolerance, a parameter of diastolic dysfunction was inversely associated with $\mathrm{HbA} 1 \mathrm{c}$ [20]. Although variations of $\mathrm{HbA1c}$ may be serve as predictors of changes in E/e' [21], in the present cohort, HbA1c was not a major predictor of annual change in E/e'. In a prior cross-sectional study, glycemic variability, determined by continuous glucose monitoring, was also shown to be associated with left ventricular diastolic function in type 2 diabetes mellitus subjects [22]. Population-based cohort study found that the presence of 
hyperinsulinemia at the baseline predicted worsening of LV diastolic dysfunction [23]. In the present study, plasma insulin was not associated with annual change in $E / e^{\prime}$ in all subjects $(r=0040, p=0.53)$, or after dividing them into diabetic $(r=0.184, p=0.11)$ and non-diabetic $(r=0.001, p=0.99)$ groups. As previously shown by our groups [24,25], visceral fat adiposity, or an adipocytokine such as leptin may be attributed to diastolic dysfunction in diabetes. Pericardial fat accumulation may also contribute to cardiac diastolic dysfunction in diabetes [26]. Another potential mediator includes presence of diabetic microangiopathy, E/e' shown to be worsened in groups classified based on albuminuria severity [27]. However, in our cohort, the presence of diabetic microangiopathy regardless of type (retinopathy, neuropathy, nephropathy) was not significantly associated with annual changes in E/e' (data not shown). Anti-diabetic agents such as the sodium glucose cotransporter type 2 (SGLT2) inhibitors may potentially affect the progression of LV diastolic dysfunction [28], which were recently shown to reduce worsening heart failure or death from cardiovascular causes in patients with heart failure [29]. Less than $5 \%$ of the present cohort was treated with SGLT2 inhibitors during registration period. Future surveys regarding antidiabetic reagents given during follow-up may unveil their potential associations with progression of LV diastolic dysfunction.

\section{Study Limitations}

This study has several limitations. First, the results of the sub-group analyses of diabetes and nondiabetes must be interpreted carefully, due to the number of subjects, potentially resulting in statistical type 1 and 2 errors. Second, not all items used as diagnostic criteria for cardiac diastolic dysfunction could be evaluated, particularly atrial volume. In several studies, E/e' has been found to lack accuracy for reflecting LV filling pressure in patients with preserved ejection fraction. Nevertheless, we consider that our results provide important initial information to unveil the pathophysiological mechanisms related to HFpEF and diabetic cardiomyopathy.

\section{Conclusions}

In patients without heart disease, sleep apnea is an important predictor for progression of LV diastolic dysfunction. Its association is partly confounded, but still independent of the presence of diabetes.

\section{Abbreviations}

AHI: apnea hypopnea index

AUC: area under the curve

A-wave: late diastolic filling velocity

BMI: Body mass index

DcT: E-wave deceleration time 
eGFR: Estimated glomerular filtration rate

E-wave: Transmitral early inflow velocity

$e^{\prime}:$ Early diastolic tissue velocity

HDL high density lipoprotein

HF: heart failure

HFpEF: heart failure with preserved ejection fraction

HSCAA: Hyogo Sleep Cardio-Autonomic Atherosclerosis

LDL: low density lipoprotein

LV: left ventricular

LVEF: LV ejection fraction

LVMI: LV mass index

ROC: receiver operating characteristic

\section{Declarations}

Ethics approval and consent to participate: The HSCAA study was approved by an appropriate institutional ethical committee (approval No. 2351) and informed written consent was obtained from each participant.

Consent for publication: not applicable

Available of data and materials: The data that support the findings of this study are available from Hyogo College of Medicine. Data are available from the authors upon reasonable request and with permission of the Hyogo College of Medicine.

Competing interests: non to declare for all authors

Funding: JSPS KAKENHI grants (19K19421 to M. Kadoya, 20K18944 to A. Morimoto, JP18K17399 to M. Kakutani-Hatayama, 19K19446 to K. Kosaka, JP18K08531 to H. Koyama), and a Hyogo College of Medicine grant (Hyogo Innovative Challenge to H. Koyama).

Authors' contributions. M.K. and H.K. contributed to conception and design, Y.K., A.M., M.K., T.D., M.K.-H., K.K.-H., A.M., K.K., Y.K., and T.S. contributed to analysis and interpretation of data, Y.K., M.K., and H.K. contributed to drafting of the article, and A.G, M.A. and M.I. provided critical revisions for important intellectual content. 
Acknowledgements: The authors are grateful for the excellent technical assistance of Sachie Koyama, Tomoe Ushitani, and Ai Matsumoto. We also wish to thank the other investigators and staff, as well as the participants of the Hyogo Sleep Cardio-Autonomic Atherosclerosis study for their valuable contributions.

\section{References}

1. Jia G, Hill MA, Sowers JR. Diabetic Cardiomyopathy: An Update of Mechanisms Contributing to This Clinical Entity. Circ Res. 2018;122(4):624-38.

2. Owan TE, Hodge DO, Herges RM, Jacobsen SJ, Roger VL, Redfield MM. Trends in prevalence and outcome of heart failure with preserved ejection fraction. N Engl J Med. 2006;355(3):251-9.

3. Lee DS, Gona P, Vasan RS, Larson MG, Benjamin EJ, Wang TJ, Tu JV, Levy D. Relation of disease pathogenesis and risk factors to heart failure with preserved or reduced ejection fraction: insights from the framingham heart study of the national heart, lung, and blood institute. Circulation. 2009;119(24):3070-7.

4. Dunlay SM, Roger VL, Redfield MM. Epidemiology of heart failure with preserved ejection fraction. Nat Rev Cardiol. 2017;14(10):591-602.

5. Resnick HE, Redline S, Shahar E, Gilpin A, Newman A, Walter R, Ewy GA, Howard BV, Punjabi NM. Sleep Heart Health S: Diabetes and sleep disturbances: findings from the Sleep Heart Health Study. Diabetes Care. 2003;26(3):702-9.

6. Wang H, Parker JD, Newton GE, Floras JS, Mak S, Chiu KL, Ruttanaumpawan P, Tomlinson G, Bradley TD. Influence of obstructive sleep apnea on mortality in patients with heart failure. J Am Coll Cardiol. 2007;49(15):1625-31.

7. Gottlieb DJ, Yenokyan G, Newman AB, O'Connor GT, Punjabi NM, Quan SF, Redline S, Resnick HE, Tong EK, Diener-West $\mathrm{M}$, et al. Prospective study of obstructive sleep apnea and incident coronary heart disease and heart failure: the sleep heart health study. Circulation. 2010;122(4):352-60.

8. Kadoya M, Koyama H, Kurajoh M, Kanzaki A, Kakutani-Hatayama M, Okazaki H, Shoji T, Moriwaki Y, Yamamoto T, Emoto $\mathrm{M}$, et al. Sleep, cardiac autonomic function, and carotid atherosclerosis in patients with cardiovascular risks: HSCAA study. Atherosclerosis. 2015;238(2):409-14.

9. Nagueh SF, Smiseth OA, Appleton CP, Byrd BF 3rd, Dokainish H, Edvardsen T, Flachskampf FA, Gillebert TC, Klein AL, Lancellotti P, et al. Recommendations for the Evaluation of Left Ventricular Diastolic Function by Echocardiography: An Update from the American Society of Echocardiography and the European Association of Cardiovascular Imaging. J Am Soc Echocardiogr. 2016;29(4):277314.

10. Diagnosis and classification of diabetes mellitus. Diabetes Care 2004, 27Suppl 1:S5-S10.

11. Morimoto A, Kadoya M, Kakutani-Hatayama M, Kosaka-Hamamoto K, Miyoshi A, Shoji T, Goda A, Asakura M, Koyama H. Subclinical decrease in cardiac autonomic and diastolic function in patients with metabolic disorders: HSCAA study. Metabolism Open. 2020;5:100025. 
12. Imai Y, Tanaka N, Usui Y, Takahashi N, Kurohane S, Takei Y, Takata Y, Yamashina A. Severe obstructive sleep apnea increases left atrial volume independently of left ventricular diastolic impairment. Sleep Breath. 2015;19(4):1249-55.

13. Wang D, Ma GS, Wang XY, Lu QQ, Wang Y, Liu NF. Left ventricular subclinical dysfunction associated with myocardial deformation changes in obstructive sleep apnea patients estimated by real-time 3D speckle-tracking echocardiography. Sleep Breath. 2016;20(1):135-44.

14. Ogilvie RP, Genuardi MV, Magnani JW, Redline S, Daviglus ML, Shah N, Kansal M, Cai J, Ramos AR, Hurwitz BE, et al. Association Between Sleep Disordered Breathing and Left Ventricular Function: A Cross-Sectional Analysis of the ECHO-SOL Ancillary Study. Circ Cardiovasc Imaging. 2020;13(5):e009074.

15. Nayor M, Enserro DM, Xanthakis V, Larson MG, Benjamin EJ, Aragam J, Mitchell GF, Vasan RS. Comorbidities and Cardiometabolic Disease: Relationship With Longitudinal Changes in Diastolic Function. JACC Heart Fail. 2018;6(4):317-25.

16. Reis JP, Allen NB, Bancks MP, Carr JJ, Lewis CE, Lima JA, Rana JS, Gidding SS, Schreiner PJ. Duration of Diabetes and Prediabetes During Adulthood and Subclinical Atherosclerosis and Cardiac Dysfunction in Middle Age: The CARDIA Study. Diabetes Care. 2018;41(4):731-8.

17. Moller JE, Pellikka PA, Hillis GS, Oh JK. Prognostic importance of diastolic function and filling pressure in patients with acute myocardial infarction. Circulation. 2006;114(5):438-44.

18. Lassen MCH, Jensen MT, Biering-Sorensen T, Mogelvang R, Fritz-Hansen T, Vilsboll T, Rossing P, Jorgensen PG. Prognostic value of ratio of transmitral early filling velocity to early diastolic strain rate in patients with Type 2 diabetes. Eur Heart J Cardiovasc Imaging. 2019;20(10):1171-8.

19. van den Hurk K, Alssema M, Kamp O, Henry RM, Stehouwer CD, Smulders YM, Nijpels G, Paulus WJ, Dekker JM. Independent associations of glucose status and arterial stiffness with left ventricular diastolic dysfunction: an 8-year follow-up of the Hoorn Study. Diabetes Care. 2012;35(6):1258-64.

20. Di Pino A, Mangiafico S, Urbano F, Scicali R, Scandura S, D'Agate V, Piro S, Tamburino C, Purrello F, Rabuazzo AM. HbA1c Identifies Subjects With Prediabetes and Subclinical Left Ventricular Diastolic Dysfunction. J Clin Endocrinol Metab. 2017;102(10):3756-64.

21. Li S, Zheng Z, Tang X, Zhong J, Liu X, Zhao Y, Chen L, Zhu J, Liu J, Chen Y. Impact of HbA1c variability on subclinical left ventricular remodeling and dysfunction in patients with type 2 diabetes mellitus. Clin Chim Acta. 2020;502:159-66.

22. Yokota S, Tanaka H, Mochizuki Y, Soga F, Yamashita K, Tanaka Y, Shono A, Suzuki M, Sumimoto K, Mukai J, et al. Association of glycemic variability with left ventricular diastolic function in type 2 diabetes mellitus. Cardiovasc Diabetol. 2019;18(1):166.

23. Cauwenberghs N, Knez J, Thijs L, Haddad F, Vanassche T, Yang WY, Wei FF, Staessen JA, Kuznetsova T. Relation of Insulin Resistance to Longitudinal Changes in Left Ventricular Structure and Function in a General Population. J Am Heart Assoc 2018, 7(7).

24. Kurajoh M, Koyama H, Kadoya M, Naka M, Miyoshi A, Kanzaki A, Kakutani-Hatayama M, Okazaki H, Shoji T, Moriwaki Y, et al. Plasma leptin level is associated with cardiac autonomic dysfunction in 
patients with type 2 diabetes: HSCAA study. Cardiovasc Diabetol. 2015;14:117.

25. Morioka T, Emoto M, Yamazaki Y, Kawano N, Imamura S, Numaguchi R, Urata H, Motoyama K, Mori $\mathrm{K}$, Fukumoto $\mathrm{S}$, et al. Leptin is associated with vascular endothelial function in overweight patients with type 2 diabetes. Cardiovasc Diabetol. 2014;13:10.

26. de Wit-Verheggen VHW, Altintas S, Spee RJM, Mihl C, van Kuijk SMJ, Wildberger JE, SchrauwenHinderling VB, Kietselaer B, van de Weijer T. Pericardial fat and its influence on cardiac diastolic function. Cardiovasc Diabetol. 2020;19(1):129.

27. Jorgensen PG, Biering-Sorensen T, Mogelvang R, Fritz-Hansen T, Vilsboll T, Rossing P, Jensen JS. Presence of micro- and macroalbuminuria and the association with cardiac mechanics in patients with type 2 diabetes. Eur Heart J Cardiovasc Imaging. 2018;19(9):1034-41.

28. Tanaka H, Soga F, Tatsumi K, Mochizuki Y, Sano H, Toki H, Matsumoto K, Shite J, Takaoka H, Doi T, et al. Positive effect of dapagliflozin on left ventricular longitudinal function for type 2 diabetic mellitus patients with chronic heart failure. Cardiovasc Diabetol. 2020;19(1):6.

29. McMurray JJV, Solomon SD, Inzucchi SE, Kober L, Kosiborod MN, Martinez FA, Ponikowski P, Sabatine MS, Anand IS, Belohlavek J, et al. Dapagliflozin in Patients with Heart Failure and Reduced Ejection Fraction. N Engl J Med. 2019;381(21):1995-2008.

\section{Tables}




\begin{tabular}{|c|c|c|c|c|}
\hline Variables & $\begin{array}{l}\text { Total } \\
(n=517)\end{array}$ & $\begin{array}{l}\text { Non-diabetes } \\
(n=342)\end{array}$ & $\begin{array}{l}\text { Diabetes } \\
(n=175)\end{array}$ & $\mathrm{P}$ \\
\hline Age, years & $58.9 \pm 0.5$ & $57.2 \pm 0.7$ & $62.2 \pm 0.8$ & $<0.01$ \\
\hline Gender, male, n (\%) & $248(48.0 \%)$ & $143(41.8 \%)$ & $105(60.0 \%)$ & $<0.01$ \\
\hline Body mass index, $\mathrm{kg} / \mathrm{m}^{2}$ & $24.4 \pm 0.2$ & $23.8 \pm 0.2$ & $25.3 \pm 0.3$ & $<0.01$ \\
\hline Current smoking, n (\%) & $119(23.0 \%)$ & $65(19.0 \%)$ & $54(30.8 \%)$ & $<0.01$ \\
\hline Systolic BP, mmHg & $125.3 \pm 0.7$ & $124.0 \pm 0.8$ & $127.7 \pm 1.2$ & 0.01 \\
\hline Diastolic BP, mmHg & $75.6 \pm 0.4$ & $75.6 \pm 0.5$ & $75.5 \pm 0.6$ & 0.93 \\
\hline Heart rate, beats/min & $68.6 \pm 0.5$ & $68.0 \pm 0.7$ & $69.6 \pm 0.9$ & 0.21 \\
\hline LDL-cholesterol, mmol/L & $2.97 \pm 0.06$ & $3.12 \pm 0.07$ & $2.70 \pm 0.09$ & $<0.01$ \\
\hline HDL-cholesterol, mmol/L & $1.43 \pm 0.02$ & $1.49 \pm 0.03$ & $1.33 \pm 0.03$ & $<0.01$ \\
\hline Triacylglycerol, mmol/L & $1.52 \pm 0.05$ & $1.43 \pm 0.06$ & $1.65 \pm 0.11$ & 0.07 \\
\hline Blood urea nitrogen, $\mathrm{mmol} / \mathrm{L}$ & $5.1 \pm 0.1$ & $5.0 \pm 0.1$ & $5.4 \pm 0.1$ & 0.06 \\
\hline Creatinine, $\mu \mathrm{mol} / \mathrm{L}$ & $63 \pm 0.9$ & $62 \pm 0.9$ & $65 \pm 1.8$ & 0.30 \\
\hline eGFR, $\mathrm{ml} / \mathrm{min} / 1.73 \mathrm{~m}^{2}$ & $83.9 \pm 1.1$ & $83.9 \pm 1.4$ & $83.8 \pm 1.9$ & 0.96 \\
\hline Fasting plasma glucose (mmol/L) & $6.1 \pm 0.1$ & $5.2 \pm 0.0$ & $7.4 \pm 0.2$ & $<0.01$ \\
\hline $\mathrm{HbA} 1 \mathrm{c}, \%$ & $6.0 \pm 0.0$ & $5.2 \pm 0.0$ & $7.3 \pm 0.1$ & $<0.01$ \\
\hline \multirow[t]{2}{*}{ Insulin (pmol/L) } & $40 \pm 1$ & $41 \pm 2$ & $38 \pm 2$ & \multirow[t]{2}{*}{0.31} \\
\hline & $(n=488)$ & $(n=342)$ & $(n=146)$ & \\
\hline \multicolumn{5}{|l|}{ Comorbidities } \\
\hline Hypertension, n (\%) & $301(64.3 \%)$ & $187(54.6 \%)$ & $114(65.1 \%)$ & 0.06 \\
\hline Dyslipidemia, n (\%) & $279(53.9 \%)$ & $165(48.2 \%)$ & $114(65.1 \%)$ & $<0.01$ \\
\hline \multicolumn{5}{|l|}{ Anti-hypertensive drugs } \\
\hline Calcium-channel blockers, n(\%) & $237(45.8 \%)$ & $163(47.6 \%)$ & $74(42.2 \%)$ & 0.26 \\
\hline ACE inhibitor or ARB, n (\%) & $132(25.5 \%)$ & $63(18.4 \%)$ & $69(39.4 \%)$ & $<0.01$ \\
\hline $\mathrm{a}$ or $\beta$ blocker, $\mathrm{n}(\%)$ & $66(12.7 \%)$ & $48(14.0 \%)$ & $18(10.3 \%)$ & 0.26 \\
\hline Diuretic agent, n (\%) & $26(5.0 \%)$ & $17(4.9 \%)$ & $9(5.1 \%)$ & 0.98 \\
\hline Statin & $138(26.7 \%)$ & $64(18.7 \%)$ & $74(42.3 \%)$ & $<0.01$ \\
\hline
\end{tabular}


Anti-diabetic drugs

DPP-4 inhibitor

GLP-1 analogue

Sulfonylurea

Thizolidine

Metformine

SGLT2 inhibitor

Insulin

Diabetes duration (years)

Diabetic microvascular complications

Neuropathy, n (\%)

Retinopathy, n (\%)

Nephropathy, n (\%)

Sleep apnea

apnea-hypopnea index (AHI)

$\mathrm{AHI}>10, \mathrm{n}(\%)$

Baseline Echocardiographic parameters

\begin{tabular}{lllll} 
LVMI & $97.5 \pm 0.9$ & $96.3 \pm 1.1$ & $100.0 \pm 1.5$ & 0.06 \\
\hline LVEF, \% & $68.7 \pm 0.3$ & $69.0 \pm 0.3$ & $68.2 \pm 0.5$ & 0.21 \\
\hline E, cm/s & $61.4 \pm 0.6$ & $62.2 \pm 0.8$ & $59.8 \pm 1.1$ & 0.10 \\
\hline A, cm/s & $69.1 \pm 0.7$ & $66.7 \pm 0.8$ & $73.9 \pm 1.1$ & $<0.01$ \\
\hline E/A & $0.93 \pm 0.0$ & $0.99 \pm 0.0$ & $0.81 \pm 0.0$ & $<0.01$ \\
\hline DcT & $209.8 \pm 2.0$ & $207.0 \pm 2.5$ & $215.4 \pm 3.6$ & 0.05 \\
\hline E/e' & $9.2 \pm 0.1$ & $9.0 \pm 2.2$ & $9.7 \pm 0.1$ & $<0.01$ \\
\hline $\mathrm{e}^{\prime}$ & $6.8 \pm 0.0$ & $7.1 \pm 0.1$ & $6.3 \pm 0.1$ & $<0.01$ \\
\hline Follow-up & & & & \\
\hline E/e'>14 event, $n(\%)$ & $77(14.9 \%)$ & $36(10.5 \%)$ & $41(23.4 \%)$ & $<0.01$ \\
\hline Annual change of E/e' & $\begin{array}{l}0.23 \pm 0.04 \\
(\mathrm{n}=306)\end{array}$ & $\begin{array}{l}0.14 \pm 0.05 \\
(\mathrm{n}=196)\end{array}$ & $\begin{array}{l}0.38 \pm 0.08 \\
(\mathrm{n}=110)\end{array}$ & 0.01 \\
& & & &
\end{tabular}

$57(32.6 \%)$

$2(1.1 \%)$

$44(25.1 \%)$

$11(6.2 \%)$

$52(29.7 \%)$

$2(1.1 \%)$

$7.0 \pm 0.5$

$62(35.4 \%)$

$10(5.7 \%)$

$37(21.1 \%)$
$29(16.5 \%)$

$\begin{array}{llll}7.0(2.9-15.2) & \begin{array}{l}5.7(2.0-12.5) \\ (n=462)\end{array} & \begin{array}{l}9.6(4.0-18.2) \\ (n=297)\end{array} & <0.01\end{array}$

$174(37.7 \%) \quad 96(32.3 \%)$

$78(47.3 \%)$ $<0.01$ 
Data are presented as the mean \pm standard error or median (25th-75th percentile) for continuous variables, and $\mathrm{n}(\%)$ for dichotomous variables. $\mathrm{P}$ values are shown comparisons of means of 2 groups (unrepeated t-test) and median (Mann-whitney test), or percentages (Chi-square test). BP, blood pressure; LDL, low density lipoprotein; HDL, high density lipoprotein; eGFR, estimated glomerular filtration rate; ACE, angiotensin converting enzyme; ARB, angiotensin II receptor blocker; DPP, dipeptidyl peptidase; GLP, glucagon-like peptide; SGLT, sodium glucose transporter; AHI, apnea hypopnea index 
Table 2. Simple linear regression analyses of the factors associated with annual progression in E/e'

\section{(A) Simple regression analyses}

\begin{tabular}{|c|c|c|}
\hline Variables & All & \\
\hline & $n=306$ & \\
\hline Age & 0.097 & \\
\hline Gender (male= 1 , female $=0$ ) & 0.047 & \\
\hline Body mass index & 0.003 & \\
\hline Current smoking, (yes=1, no=0) & 0.024 & \\
\hline Diabetes mellitus (yes= $1, \mathrm{no}=0$ ) & 0.147 & * \\
\hline Hypertension & 0.101 & \\
\hline Dyslipidemia & 0.038 & \\
\hline Systolic blood pressure & 0.035 & \\
\hline Diastolic blood pressure & -0.068 & \\
\hline Heart rate & 0.057 & \\
\hline low density lipoprotein-cholesterol & 0.099 & \\
\hline high density lipoprotein-cholesterol & -0.065 & \\
\hline Triacylglycerol & 0.092 & \\
\hline Blood urea nitrogen & 0.117 & \\
\hline Creatinine & 0.015 & \\
\hline estimated glomerular filtration rate & -0.016 & \\
\hline Fasting plasma glucose & 0.050 & \\
\hline $\mathrm{HbA1c}$ & 0.101 & \\
\hline \multirow[t]{2}{*}{ Insulin } & 0.040 & \\
\hline & $(n=286)$ & \\
\hline Baseline E/e' & -0.264 & ** \\
\hline In apnea-hypopnea index & 0.128 & * \\
\hline \multicolumn{3}{|l|}{ Anti-hypertensive drugs } \\
\hline Calcium-channel blockers & 0.023 & \\
\hline Inhibitors of renin-angiotensin system & 0.129 & * \\
\hline
\end{tabular}


(B) Multiple regression analyses

$\begin{array}{lll} & \text { All } & \\ & \mathrm{n}=306 & * * \\ \text { Diabetes }(\mathrm{yes}=1, \mathrm{no}=0) & 0.278 & * * \\ \text { In apnea-hypopnea index } & 0.138 & * * \\ \mathrm{R}^{2} & 0.140\end{array}$

(A) Pearson's correlation coefficients are shown. ${ }^{*} p<0.05, * * p<0.01$.

(B) Standard regression coefficients are shown. All analyses include age, gender, BMI, hypertension, dyslipidemia and baseline E/e' as covariates. ${ }^{*} p<0.05$, ${ }^{\star \star} p<0.01$.

Figures 


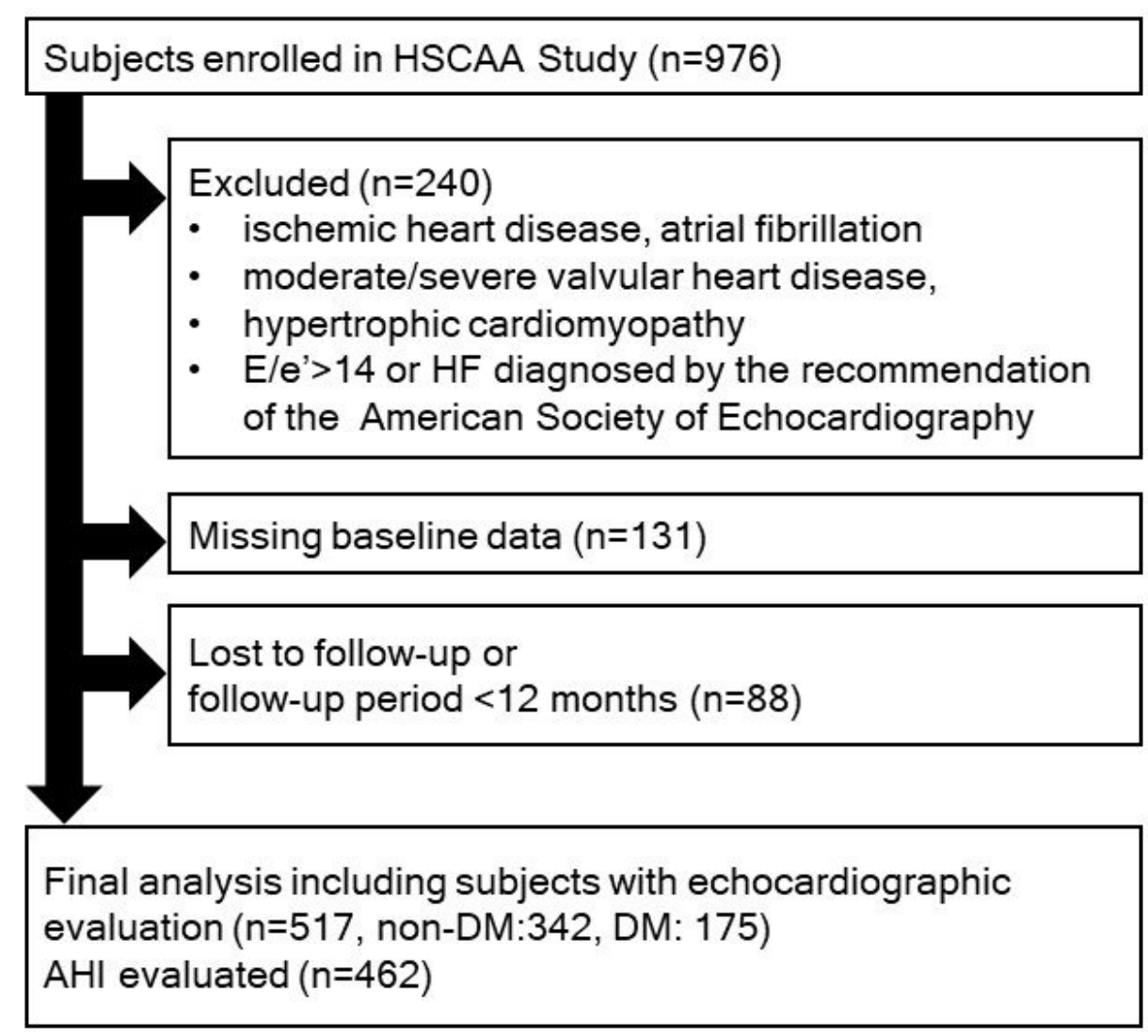

Figure 1

Figure 1

Study participants. 


\section{A) All subjects}

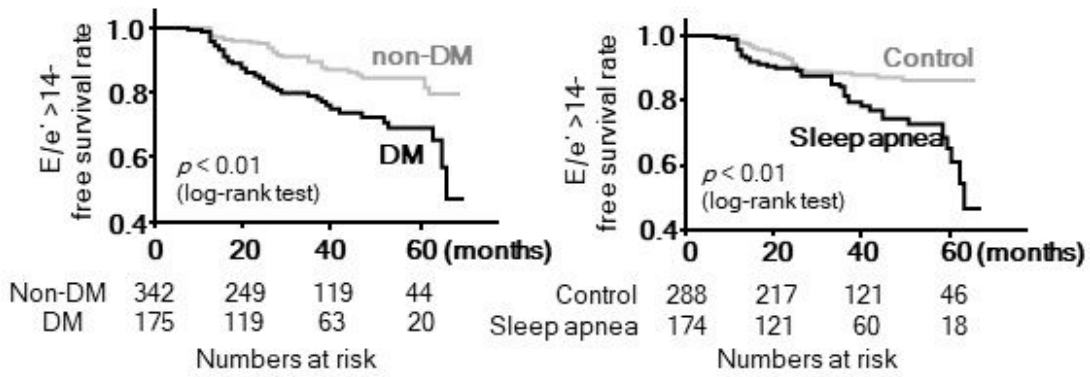

B) Non-diabetic subjects

\section{C) Diabetic subjects}

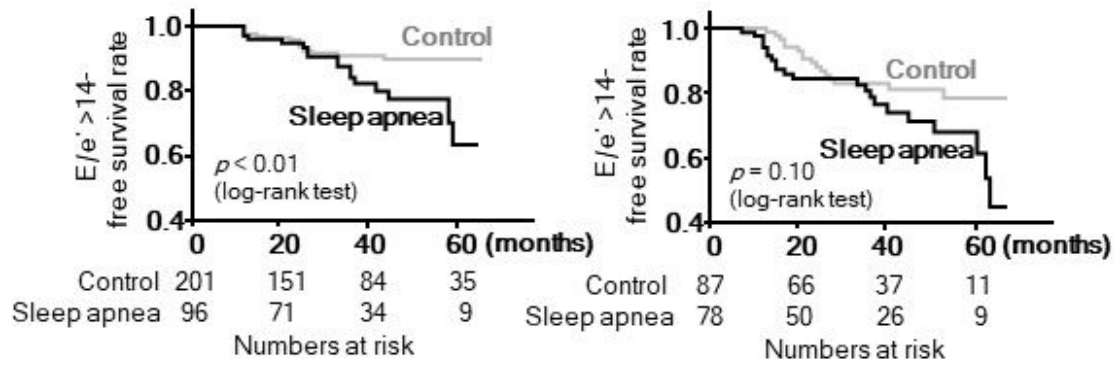

Figure 2

\section{Figure 2}

Kaplan Meier analysis of effects of diabetes and sleep apnea on cardiac diastolic dysfunction as defined by $E / e^{\prime}>14$ in all (A), non-diabetic (B), and diabetic subjects (C). Sleep apnea was defined based on apnea-hypopnea index $>10$. 

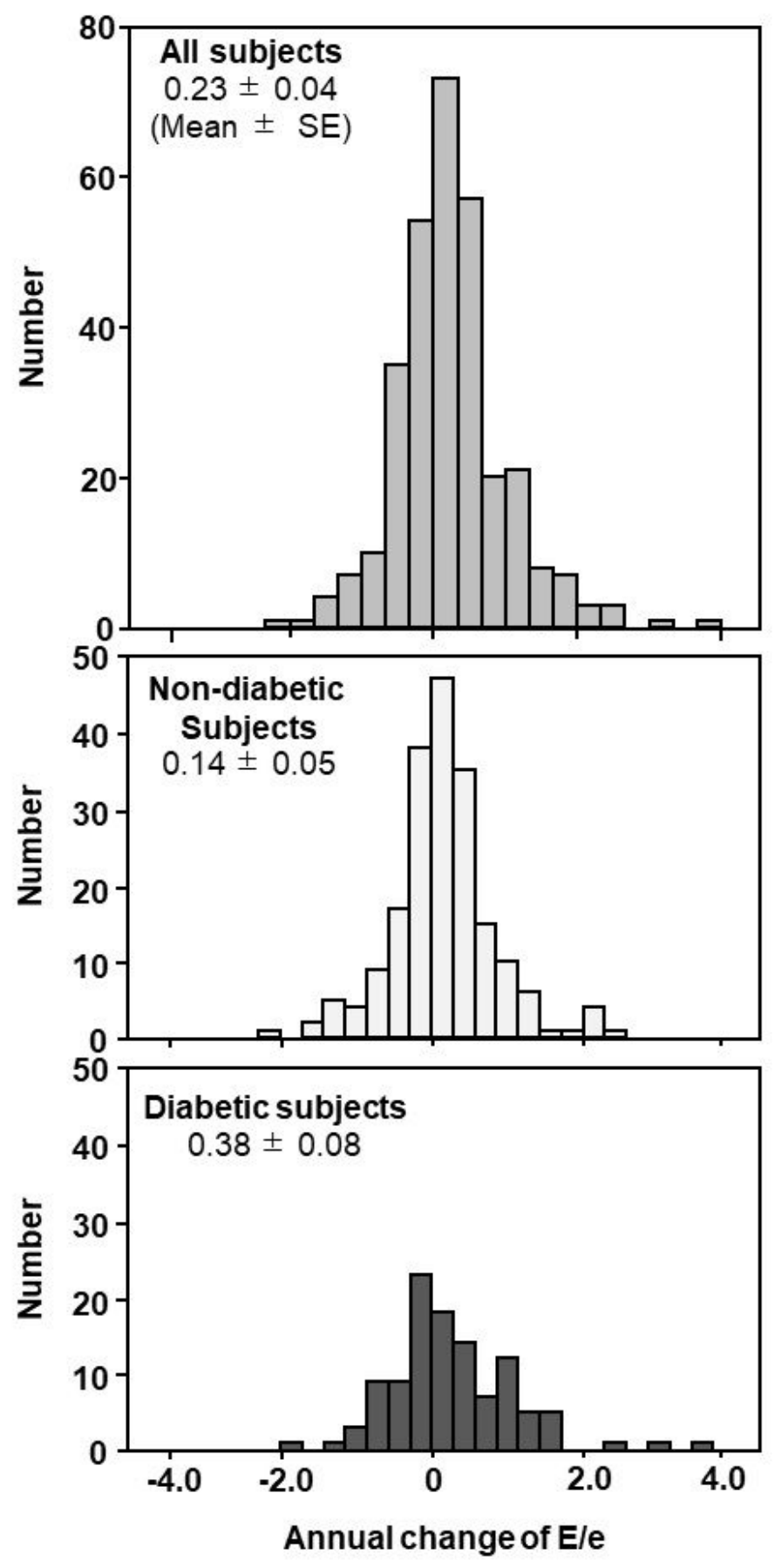

Figure 3

Figure 3

Distribution of subjects (all, non-diabetic, and diabetic subjects) according to annual changes in E/e'. 

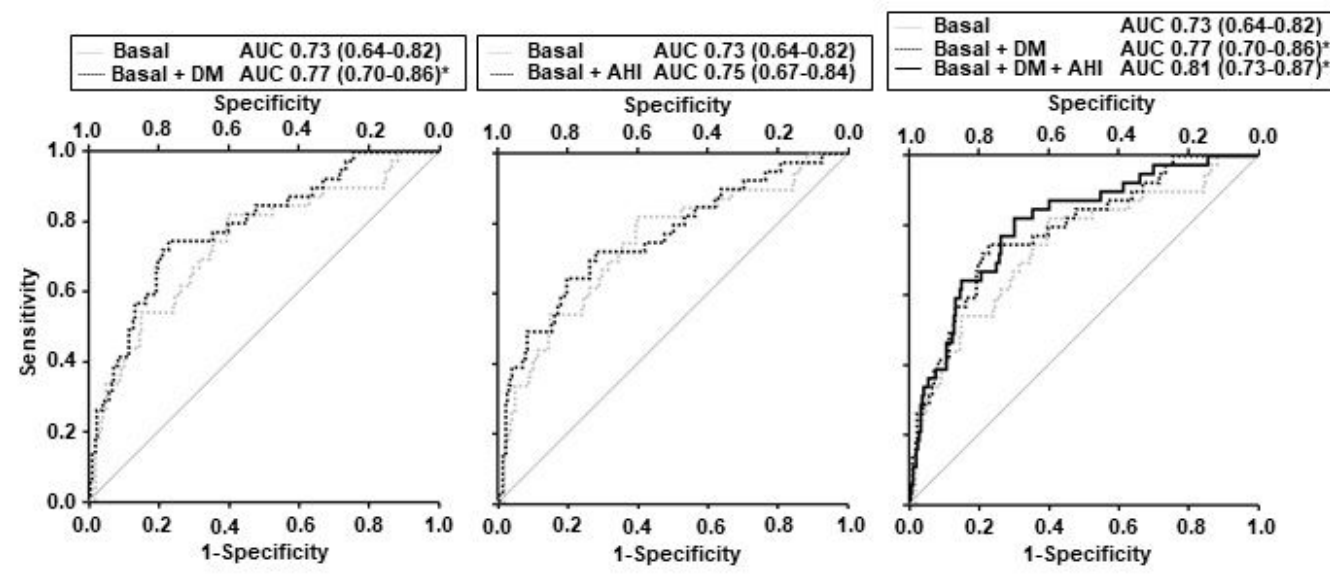

Figure 4

\section{Figure 4}

Receiver operating characteristics curves and area under the curve for predicting subjects with annual change of $E / \mathrm{e}^{\prime}>1.0$. The figures show results of analysis of the impact of addition of diabetes, $\mathrm{AHI}$, and diabetes $+\mathrm{AHI}$ to the basal models (age, gender, BMI, hypertension, dyslipidemia, baseline E/e'). ${ }^{*} p<0.05$ vs basal model. 